\title{
Independent and epistatic effects of variants in VPS10-d receptors on Alzheimer disease risk and processing of the amyloid precursor protein (APP)
}

\author{
C Reitz ${ }^{1,2,3}$, G Tosto ${ }^{2}$, B Vardarajan ${ }^{4}$, E Rogaeva ${ }^{5}$, M Ghani ${ }^{5}$, RS Rogers ${ }^{2}$, C Conrad ${ }^{2}$, JL Haines ${ }^{6}$, MA Pericak-Vance ${ }^{7}$, MD Fallin ${ }^{8}$, \\ T Foroud $^{9}$, LA Farrer ${ }^{4,10,11,12,13,14}$, GD Schellenberg ${ }^{15}$, PS George-Hyslop ${ }^{5,16,17}$, R Mayeux ${ }^{1,2,3,18,19,20}$ and the Alzheimer's Disease \\ Genetics Consortium (ADGC)
}

Genetic variants in the sortilin-related receptor (SORL1) and the sortilin-related vacuolar protein sorting 10 (VPS10) domaincontaining receptor 1 (SORCS1) are associated with increased risk of Alzheimer's disease (AD), declining cognitive function and altered amyloid precursor protein (APP) processing. We explored whether other members of the (VPS10) domain-containing receptor protein family (the sortilin-related VPS10 domain-containing receptors 2 and 3 (SORCS2 and SORCS3) and sortilin (SORT1)) would have similar effects either independently or together. We conducted the analyses in a large Caucasian case control data set ( $n=11840$ cases, 10931 controls) to determine the associations between single nucleotide polymorphisms (SNPs) in all the five homologous genes and AD risk. Evidence for interactions between SNPs in the five VPS10 domain receptor family genes was determined in epistatic statistical models. We also compared expression levels of SORCS2, SORCS3 and SORT1 in AD and control brains using microarray gene expression analyses and assessed the effects of these genes on $\gamma$-secretase processing of APP. Several SNPs in SORL1, SORCS1, SORCS2 and SORCS3 were associated with AD. In addition, four specific linkage disequilibrium blocks in SORCS1, SORCS2 and SORCS3 showed additive epistatic effects on the risk of $A D(P \leqslant 0.0006)$. SORCS3, but not SORCS2 or SORT1, showed reduced expression in AD compared with control brains, but knockdown of all the three genes using short hairpin RNAs in HEK293 cells caused a significant threefold increase in APP processing (from $P<0.001$ to $P<0.05$ ). These findings indicate that in addition to SORL1 and SORCS1, variants in other members of the VPS10 domain receptor family (that is, SORCS1, SORCS2, SORCS3) are associated with AD risk and alter APP processing. More importantly, the results indicate that variants within these genes have epistatic effects on AD risk.

Translational Psychiatry (2013) 3, e256; doi:10.1038/tp.2013.13; published online 14 May 2013

\section{Introduction}

A central event in the pathogenesis of Alzheimer's disease $(A D)$ is the deposition of amyloid $\beta(A \beta)$ 1-40 and $A \beta 1-42$ peptides generated by proteolytic cleavage by $\beta$ - and $\gamma$-secretase from a larger membrane-bound protein, the amyloid precursor protein (APP). ${ }^{1}$ APP and the secretases are integral transmembrane proteins dynamically sorted through the plasma membrane. Modulation of APP sorting through the membrane or altering APP cleavage by secretase enzymes could affect the regulation of $A \beta$ production or processing.

\begin{abstract}
Variants in two members of the vacuolar protein sorting 10 (VPS10) domain-containing receptor protein family, sortilinrelated receptor (SORL1) and sortilin-related VPS10 domaincontaining receptor 1 (SORCS1), are associated with lateonset $A D$ presumably through effects on APP sorting and cleavage. $^{2-4}$ The VPS10 domain-containing receptor protein family contains five type I membrane homologs (SORL1, sortilin (SORT1), SorCS1, SorCS2 and SorCS3), ${ }^{5-9}$ that are expressed in the central nervous system. All contain a single Vps10p-D situated at the N-terminus of their luminal/extracellular moiety. The VPS10 motif functions as a sorting
\end{abstract}

\footnotetext{
${ }^{1}$ The Taub Institute for Research on Alzheimer's Disease and the Aging Brain, Columbia University, New York, NY, USA; ${ }^{2}$ The Gertrude H. Sergievsky Center, College of Physicians and Surgeons, Columbia University, New York, NY, USA $;{ }^{3}$ Department of Neurology, College of Physicians and Surgeons, Columbia University, New York, NY, USA; ${ }^{4}$ Department of Biostatistics, Boston University Schools of Medicine and Public Health, Boston, MA, USA; ${ }^{5}$ Centre for Research in Neurodegenerative Diseases, University of Toronto, Ontario, Canada; ${ }^{6}$ Center for Human Genetics Research and Department of Molecular Physiology and Biophysics, Vanderbilt University Medical Center, Nashville, TN, USA; ${ }^{7}$ Miami Institute for Human Genomics, University of Miami, Miller School of Medicine, Miami, FL, USA; ${ }^{8}$ Johns Hopkins Bloomberg School of Public Health, Johns Hopkins University, Baltimore, MD, USA; ${ }^{9}$ Department of Medical and Molecular Genetics, Indiana University, Indianapolis, IN, USA; ${ }^{10}$ Department of Medicine (Biomedical Genetics), Boston University Schools of Medicine and Public Health, Boston, MA, USA; ${ }^{11}$ Department of Neurology, Boston University Schools of Medicine and Public Health, Boston, MA, USA; ${ }^{12}$ Department of Ophthalmology, Boston University Schools of Medicine and Public Health, Boston, MA, USA; ${ }^{13}$ Department of Genetics and Genomics, Boston University Schools of Medicine and Public Health, Boston, MA, USA; ${ }^{14}$ Department of Epidemiology, Boston University Schools of Medicine and Public Health, Boston, MA, USA; ${ }^{15}$ Department of Pathology and Laboratory Medicine, University of Pennsylvania School of Medicine, Philadelphia, PA, USA; ${ }^{16}$ Department of Medicine, University Health Network, Toronto, Ontario, Canada; ${ }^{17}$ Department of Clinical Neurosciences, Cambridge Institute for Medical Research, University of Cambridge, Cambridge, UK; ${ }^{18}$ Department of Psychiatry, College of Physicians and Surgeons, Columbia University, New York, NY, USA; ${ }^{19}$ Department of Medicine, College of Physicians and Surgeons, Columbia University, New York, NY, USA and ${ }^{20}$ Department of Epidemiology, School of Public Health, Columbia University, New York, NY, USA

Correspondence: Dr R Mayeux, Gertrude H. Sergievsky Center, Columbia University, 630 West 168th Street, New York, NY 10032, USA.

E-mail: rpm2@ columbia.edu
}

Keywords: Alzheimer's disease; SORCS2; SORCS3; SORT1

Received 2 October 2012; revised 6 Decemeber 2012; accepted 3 January 2013 
receptor in the Golgi compartment required for the intracellular sorting and delivery of proteins, including APP. In SORT1, also known as neurotensin receptor-3, the Vps10p-D makes up the entire luminal extracellular part of the receptor, but the other four receptors have additional modules. In SORL1, the Vps10p-D is followed by five lowdensity lipoprotein receptor class $B$ repeats flanked by an epidermal growth factor precursor-type repeat, a cluster of 11 low-density lipoprotein receptor class A repeats and 6 fibronectin type-III repeats. The mutually highly homologous SorCS1, SorCS2 and SorCS3 contain a leucine-rich segment between the Vps10p-D and the transmembrane domain. Structure prediction of the leucine-rich segment suggests a beta-sandwich fold and relates the domain to the immunoglobulin-like fold (E-set) superfamily. Following the extracellular and transmembrane segment, each receptor carries a short (40-80 amino acids) cytoplasmic domain comprising typical motifs for interaction with cytosolic adaptor molecules. In genomic DNA, members of this family are large with many exons but the coding sequence lengths are usually $<3700$ nucleotides. Very large introns (introns 1-2) typically separate the exons encoding the VPS10 domain; the remaining exons are separated by much smaller introns. Exons $1-3$ encode the VPS10 domain.

Previously, we demonstrated that SORL1 modulates the translocation and retention of APP in subcellular compartments, which are less favorable for secretase processing, thereby reducing the extent of proteolytic breakdown into both amyloidogenic and non-amyloidogenic products. ${ }^{3}$ Furthermore, we showed that under-expression of SORL1 leads to overexpression of $A \beta$ and an increased risk of AD. Subsequently, we demonstrated that genetic variation in SORCS1 also influences $A D$ risk, cognitive performance, APP processing and $A \beta 40$ and $A \beta 42$ levels through an effect on $\gamma$-secretase processing of APP. ${ }^{2,10}$ Overexpression of SorCS1 reduced $A \beta 40$ and $A \beta 42$ levels, whereas suppression of SorCS1 increased $\gamma$-secretase processing of APP. The association of SORL 1 with AD has been supported by a metaanalysis of Caucasian and Asian data sets that included a total of 12464 cases and 17929 controls $^{11}$ and has been further validated in various ethnic groups, including African Americans, Israeli Arabs and Caribbean Hispanics, although with some degree of allelic heterogeneity. ${ }^{3,11-18}$ In addition, these data are supported by a study in which overexpression of SorCS1c $\beta$-myc in cultured cells caused a significant reduction in $A \beta$ generation, whereas, conversely, endogenous murine $A ß 40$ and $A ß 42$ levels were increased in the brains of Sorcs1 hypomorphic mice. ${ }^{19}$

We hypothesized that variants in other members of the sortilin-related VPS10 domain containing receptor family, namely SORCS2, SORCS3 and SORT1, would also be associated with $A D$ risk either independently or through epistatic effects. These homologous genes are expressed in different brain regions with different subcellular localisations, ${ }^{20-22}$ but there are many brain regions, such as the hippocampus, in which these genes are co-expressed albeit at low levels. ${ }^{20}$

We conducted single-marker association and epistasis analyses of all the five homologous genes in a large Caucasian case-control data set, with sufficient power to detect modest effect sizes and interactive effects. In addition, we conducted microarray gene expression analyses and $\gamma$-secretase assays for SORCS1, SORCS2, SORCS3 and SORT1.

\section{Participants and methods}

Ethics statement. Informed consent was obtained from all the participants using procedures approved by institutional review boards at each of the clinical research centers collecting human subjects for the ADGC project.

Participants. The data set included 11840 cases and 10931 controls from the ADGC data set. ${ }^{23}$ The clinical characteristics are summarized in Table 1. The diagnoses of 'probable' or 'possible' AD were defined based on the National Institute of Neurological and Communication Disorders and Stroke-Alzheimer's Disease and Related Disorders Association (NINCDS-ADRDA) diagnosis criteria at clinics specializing in memory disorders or in clinical investigations. Persons were classified as 'controls' when they were without cognitive impairment or dementia at last visit.

Genotyping. HapMap2-imputed genotypic data for single nucleotide polymorphisms (SNPS) in SORCS1 $(n=648)$, SORCS2 $(n=740)$, SORCS3 $(n=742)$, SORL1 $(n=160)$ and SORT1 $(n=40)$ was obtained from the previously published genome-wide association study. ${ }^{23}$ The SNPs assessed included both intronic and exonic SNPs. The SORCS1 SNPs were not identical to the SNPs assessed in our previous study, which had been selected based on previous reports. ${ }^{2}$ Details regarding apolipoprotein $\mathrm{E}$ (APOE) genotyping are described in the Supplementary methods.

Cell culture and transfection. Using HEK293 cell lines, reverse transcriptase-PCR (RT-PCR) and western analysis were used to detect all five VPS10 proteins and to verify the knockdown and specificity of each short hairpin RNA (shRNA) as previously described. ${ }^{2}$ The corresponding shRNA DNA sequences are shown in Supplementary Table 3.

APP-GV Assay. The $\gamma$-secretase activity and nuclear translocation of the APP/Fe65/TIP60 protein complex was

Table 1 Characteristics of the study sample

\section{Characteristics}

\begin{tabular}{lc}
\hline Number of cases with AD & 11840 \\
Number of controls & 10931 \\
Age at Onset for AD cases (s.d.) & $74.55(6.8)$ \\
Age at last exam for controls (s.d.) & $76.26(7.2)$ \\
Proportion of females & $59.66 \%$ \\
& \\
Frequency of APOE e4+ & \\
Cases & 0.38 \\
Controls & 0.14 \\
Frequency of APOE e4 - & \\
Cases & \\
Controls & 0.62 \\
\hline
\end{tabular}

Abbreviation: AD, Alzheimer's disease; APOE, apolipoprotein E. 
monitored with the APP-GV assay. ${ }^{24}$ The APP-GV assay is a luciferase-based assay $^{24}$ consisting of the APP gene's C-terminus (AICD) fused to a transcription factor composed of the GAL4 DNA-binding domain with VP16 transcriptional activator (GV). In addition, the AICD fragment is fused to the GV domains as a positive control of AICD generation and allows for the evaluation of the AICD-specific contribution to the observed modulation in the APP-GV assay. Briefly, SorCS2 cDNA or SorCS2 shRNAs transiently transfected were evaluated in either the APP-GV or the AICD-GV assay, as previously described ${ }^{24}$ in the HEK293 cell line. SorCS3 cDNA or SorCS3 shRNAs, and SORT1 cDNA or SORT1 shRNAs, were evaluated in a similar fashion.

Microarray gene expression and quantitative RT-PCR. Expression profiling was performed separately for the cerebellum, parietal-occipital neocortex and amygdala regions from $19 A D$ and 10 control brains from the New York Brain Bank (www.nybb.hs.columbia.edu). This threeregion approach allowed us to enhance the signal-to-noise ratio $^{25}$ and to determine those changes in expression that are specific for late-onset $A D$ and consistent with the distribution of $A D$ pathology. For the expression profiling of $A D$ and control brains, the Affymetrix GeneChip Human Exon 1.0 ST Arrays (Affymetrix, Santa Clara, CA, USA) were used. Frozen brain tissue was ground over liquid nitrogen and stored at $-80^{\circ} \mathrm{C}$ until use. Total RNA was extracted and purified using the TRIzol Plus RNA purification kit (Invitrogen, Life Technologies, Grand Island, NY, USA). All RNA preparations were analyzed using an Agilent Bioanalyzer (Agilent Technologies, Santa Clara, CA, USA; RNA 6000 nano-kit) to determine RNAquantity/quality and only samples with RNA integrity number $>8$ were used in the subsequent RNA amplification and hybridization steps. The Genechip expression two-cycle target labeling kit (Affymetrix) was used for all samples according to Affymetrix's protocols. Briefly, the procedure consists of an initial ribosomal RNA reduction step and two cycles of reverse transcription followed by in vitro transcription. For each sample, $1 \mu \mathrm{g}$ of total RNA is initially subjected to removal of ribosomal RNA using the RiboMinus Transcriptome Isolation Kit (Invitrogen) and spiked with Eukaryotic PolyA RNA controls (Affymetrix). The ribosomal RNA-depleted fraction was used for cDNA synthesis by reverse transcription primed with T7-random hexamer primers, followed by second strand synthesis. This cDNA served as the template for in vitro transcription to obtain amplified antisense cRNA. Subsequently, cRNA from the first round was reverse transcribed using random primers to obtain single-stranded sense DNA. In this second reverse transcription, dUTP (2'-deoxyuridine, 5 '-triphosphate) is incorporated into the DNA to allow for subsequent enzymatic fragmentation using a combination of UDG (uracil-DNA glycosylase) and APE1 (apyrimidinic endonuclease 1). All reverse and in vitro transcription steps were performed using the GeneChip WT cDNA synthesis and amplification Kit (Affymetrix). The resulting fragmented DNA was labeled with Affymetrix DNA Labeling Reagent. Labeled fragmented DNA was hybridized to Affymetrix Human Exon 1.0 ST arrays, washed and stained using the GeneChip Hybridization, Wash and Stain Kit (Affymetrix).
Fluorescent images were recorded on a Genechip scanner 3000 and analyzed with the GeneChip operating software.

Significant results obtained from the microarray study were validated by quantitative RT-PCR using the same set of $A D$ and control samples. Total RNA $(1 \mu \mathrm{g})$ from of the amygdala region was used to generate cDNAs using the AffinityScript first-strand synthesis kit (Agilent Stratagene, CA, USA). RT-PCR primers were designed for three randomly selected exons of SorCS3 (10, 17 and 21). The housekeeping gene, TBP (TATA-binding protein), was used as the endogenous control; and samples were analyzed in triplicate. The primers used in the quantitative RT-PCR are available from Supplementary Table 1. Real-time RT-PCR was done using SYBR Green reagent (TaKaRa Mirus Bio, Madison, WI, USA) on an ABI7500 system (Applied Biosystems, Foster City, CA, USA).

Statistical methods. Extensive quality review of SNPs and samples were previously completed. ${ }^{23}$ Then multivariate logistic regression analyses in PLINK (http://pngu.mgh. harvard.edu/ purcell/plink/) were used to assess additive genotypic and allelic associations with $A D$ risk in the casecontrol data sets, and generalized estimating equation models were used for family-based data sets. All models were first adjusted for age at examination, sex and population stratification and subsequently for $A P O E-\varepsilon 4$ (additive effect) as well. For adjustment for population stratification, the first two, three or four estimated principal components were used, as described previously. ${ }^{23}$ Logistic generalized estimating equation models ${ }^{26,27}$ were used to evaluate association in the family-based data sets, using the same adjustments. Then, a meta-analysis of the individual study results was performed using inverse variance weights for the effect estimates as implemented in METAL (http:// www.sph.umich.edu/csg/abecasis/Metal/). In order to take linkage disequilibrium (LD) between the markers into account, the $P$-value threshold for multiple testing correction was, in both single-marker and epistasis analyses, determined by applying the algorithm by $\mathrm{Li}$ and $\mathrm{Ji}^{28}$ As this was a candidate gene study with the a priori hypothesis of an association between each of the explored genes and $A D$, the calculation was done separately for each gene.

Epistasis. Using only the SNPs that were associated with $\mathrm{AD}$ in the single-marker analyses $(P \leqslant 0.05)$, we tested for an interaction between SNPs in the five homologous genes. The analysis was carried out using PLINK (http://pngu.mgh. harvard.edu/ purcell/plink/) adjusting for population stratification. The model based on generalized estimating equations yields a list of SNP-by-SNP comparisons with beta coefficients and $P$-values. Based on the number of independent interactions tested, we accepted a $P$-value of $\leqslant 0.0001$ as statistically significant. As described above, LD between the markers was taken into account applying the algorithm by $\mathrm{Li}$ and $\mathrm{Ji}^{28}$

Statistical analysis for the gene expression and quantitative RT-PCR data. To determine in which genes expression levels differ between affected and unaffected brain regions, as well as between $A D$ and control brains, 
4

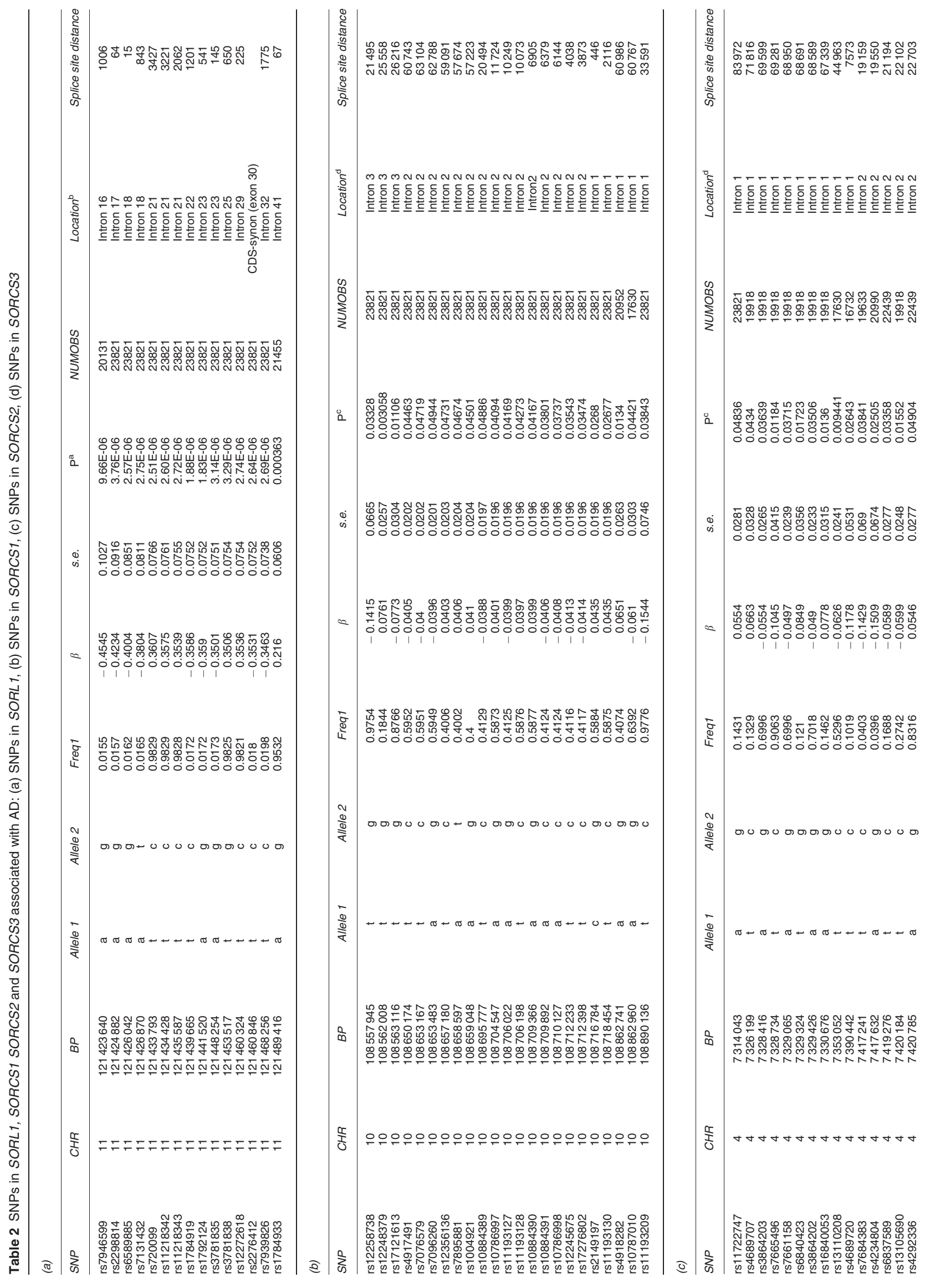




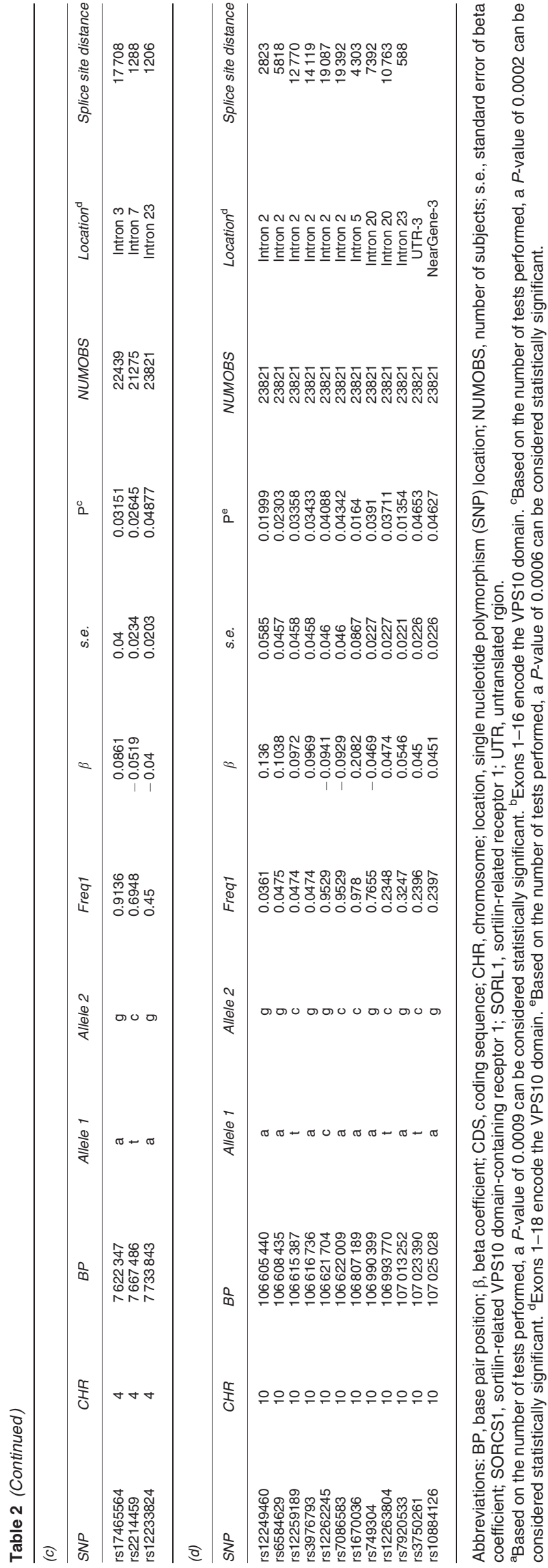

we performed both within- and between-group factors' analysis of variance using PARTEK GENOMICS SUITE 6.4 (http://www.partek.com/partekgs). Before the expression analysis, we $\log _{10}$-transformed the Rank invariant normalized expression data. False discovery rate was used to account for the error in multiple comparisons. The real-time RT-PCR data were analyzed by the comparative CT method integrated in the DataAssist Software (Life Technologies). ${ }^{29}$

Statistical analysis for the cell biology assays. Mean expression levels were compared by analysis of variance with post hoc correction using Graphpad Statistical software (Graphpad, Inc., San Diego, CA, USA). All data were normalized to transfection efficiency (for example, green fluorescent protein) and then to the control values on each plate for every assay to allow for comparisons across experiments.

\section{Results}

Single-marker analyses. Table 1 shows the characteristics of the study populations. In all, 15 SNPs in SORL1, 23 SNPs in SORCS1, 18 SNPs in SORCS2 and 12 SNPs in SORCS3 were associated with AD (Table 2). These SNPs belonged to distinct LD blocks in these genes (Supplementary Figure S1). All SNPS in SORL1 reached statistical significance after correction for multiple testing and taking LD between the markers into account. One of these SNPs, rs1784933, corresponds to SNP26 in the original study by Rogaeva et al. ${ }^{3}$ and is located $6 \mathrm{~kb}$ from SNP 25, which is part of one of the two SORL1 clusters that have been repeatedly associated with $A D$ in different ethnic groups., ${ }^{3,18}$ The SNPs in SORCS1, SORCS2 and SORCS3 were close, but not statistically significant. Interestingly, in line with previous reports, ${ }^{2}$ most of the significant SNPs in SORCS1 and SORCS2 are located in intron 1, which is adjacent to the exons encoding the VPS10 domain. In addition, in all the four homologs (SORL1, SORCS1, SORCS2, SORCS3) some of the disease-associated SNPs were close to splice sites (Table 2). None of the genotyped SNPs in SORT1 were significantly associated with AD (Supplementary Table 2).

Epistasis analysis. Upon testing for epistatic effects between the SNPs that were associated in the single-marker analyses (Table 3a), 34 pairs of SNPs showed epistatic effects at a $P$-value of $<0.01$. The vast majority ( $n=26$ pairs) included a specific LD block in SORCS3 with two specific LD blocks in SORCS2 (Table 3, Figures 1a and b). Consistent with the single-marker analyses, the interacting SNPs were located in introns 1 and 2 (Table 3, Figure 1), adjacent to the exons coding for the VPS10 domain. The epistasis $\beta$ for these SORCS2/SORCS3 interactions ranged from -0.94 to 0.94 , reflecting larger effects (additive) than the singlemarker effects $(-0.15<\beta<0.20)$.

Eight pairs resembled additional epistatic effects between SORCS1/SORCS3 and between SORCS1/SORCS2 (Table 3b). Of note, the single SORCS3 SNP (rs1670036) interacting with SORCS1 is located in the specific LD block also showing interaction with SORCS2 (Figure 1a), and again all SNPs constituting the LD block in SORCS1 are located in 
赵

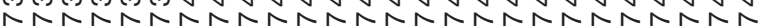

โั จำ

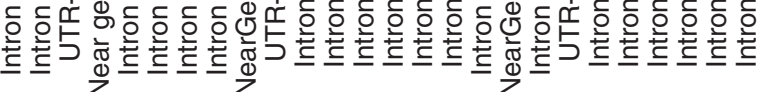

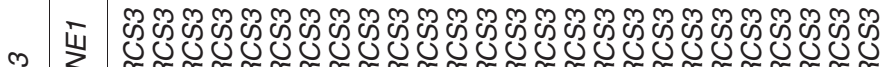
0
0

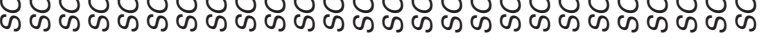

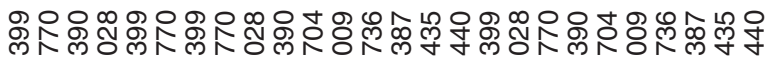

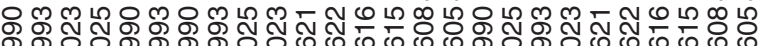

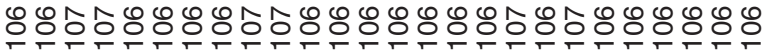
œ

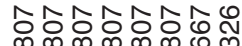
8888881

$\frac{0}{\frac{1}{0}}$

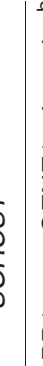

s. 으으으으으으하 nn-nnn-m

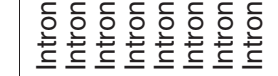

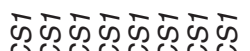

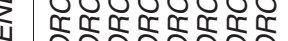

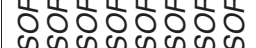

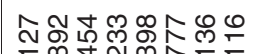
i

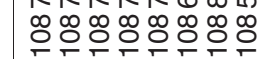

$\frac{\pi}{\frac{\pi}{3}}$ 으우우으우으으우 $1|1|+{ }^{++}|1|+|1| 1+1+++1++++1+1 \mid$

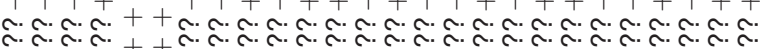
$|1| 1+++1|+| 1 \mid 1+1+1+1++++1+1+$ $1|1|++1|+1| 1 \mid+1+++1++++1+11$ $111+++11+111+1+1++1+++1+1+11$

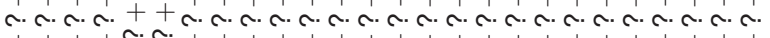

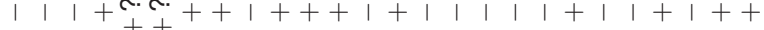

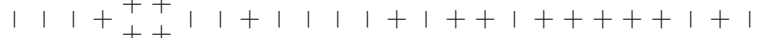
$1\left|1+{ }^{1}+1\right|+111+1+++1++++1+11$

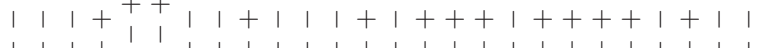

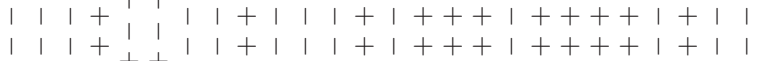
लํำ

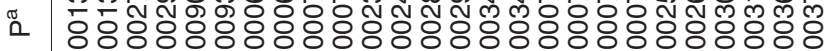
00000000000000000000000000

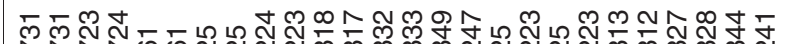

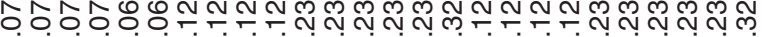
00000000000000000000000000

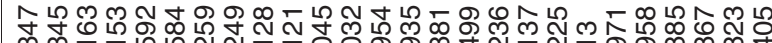

岳

岗

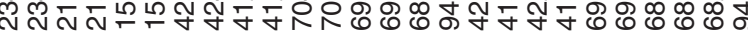
00000000000000000000000000 ชั $\bar{\varangle}$

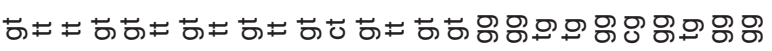

के

(1) क S. ब. 0 क

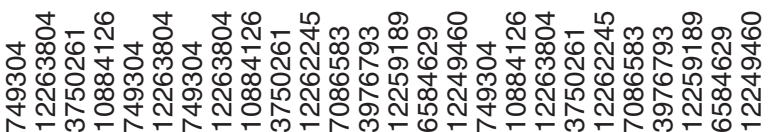

$\therefore$

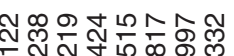

ㄴํㅇㅇํㅇㅇㅇㅇㅇㅇㅇํㅇ 0.0000000

ब.ं.

옹용Nㅡ음

is

占

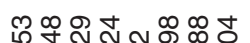

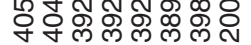
00000000

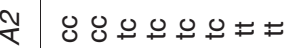
000 OOOOOON

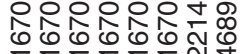
क ㄷㅇ유요욤요 이의 i 
a

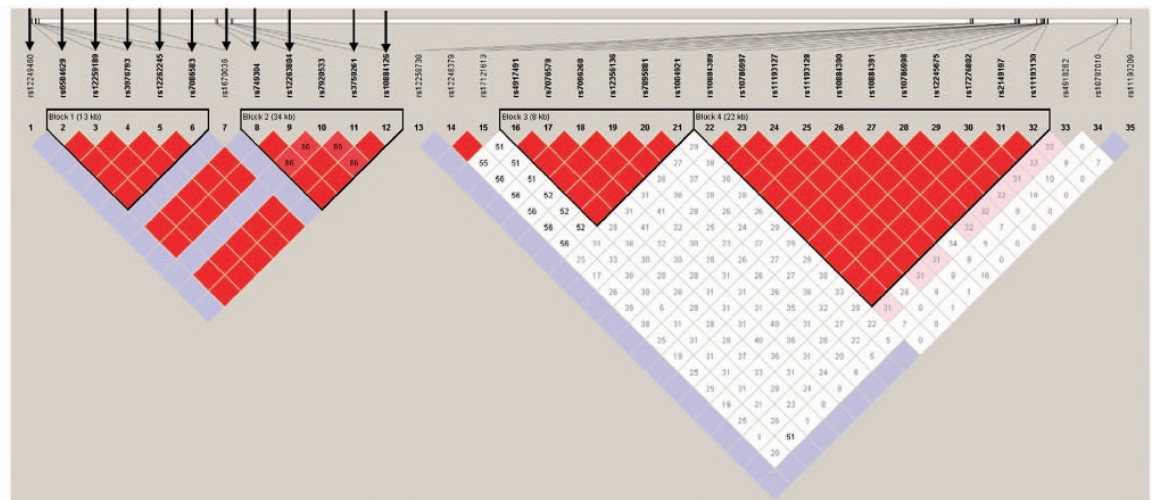

b

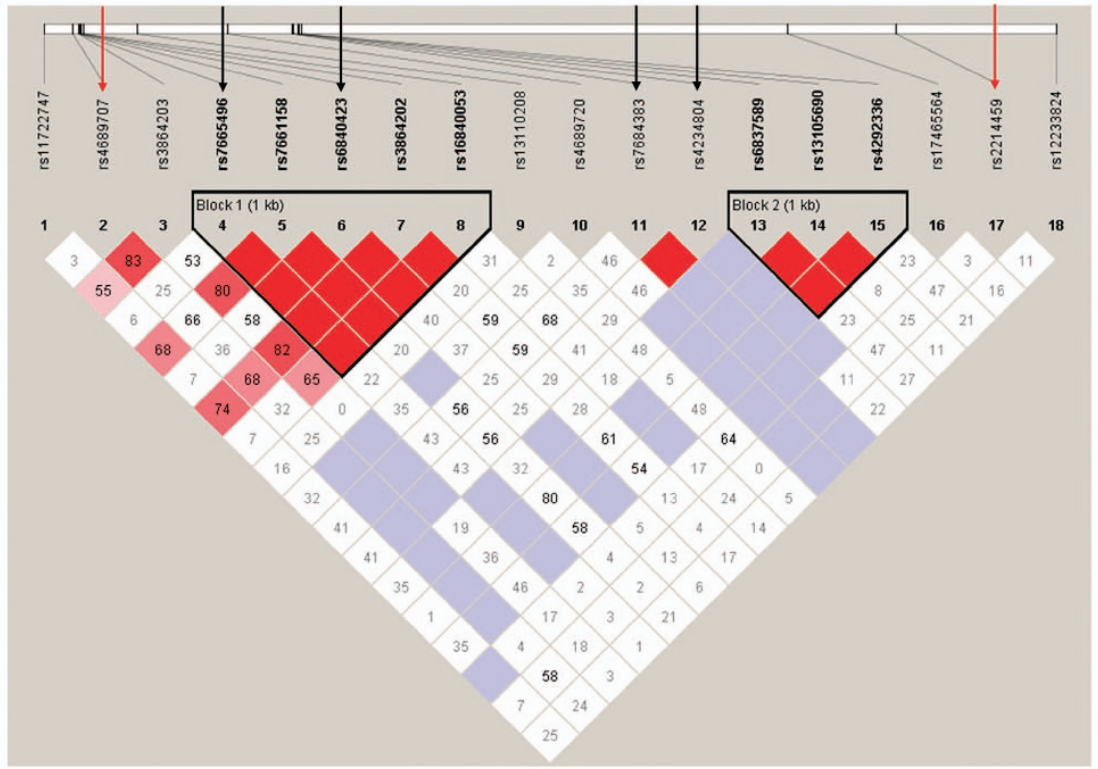

c

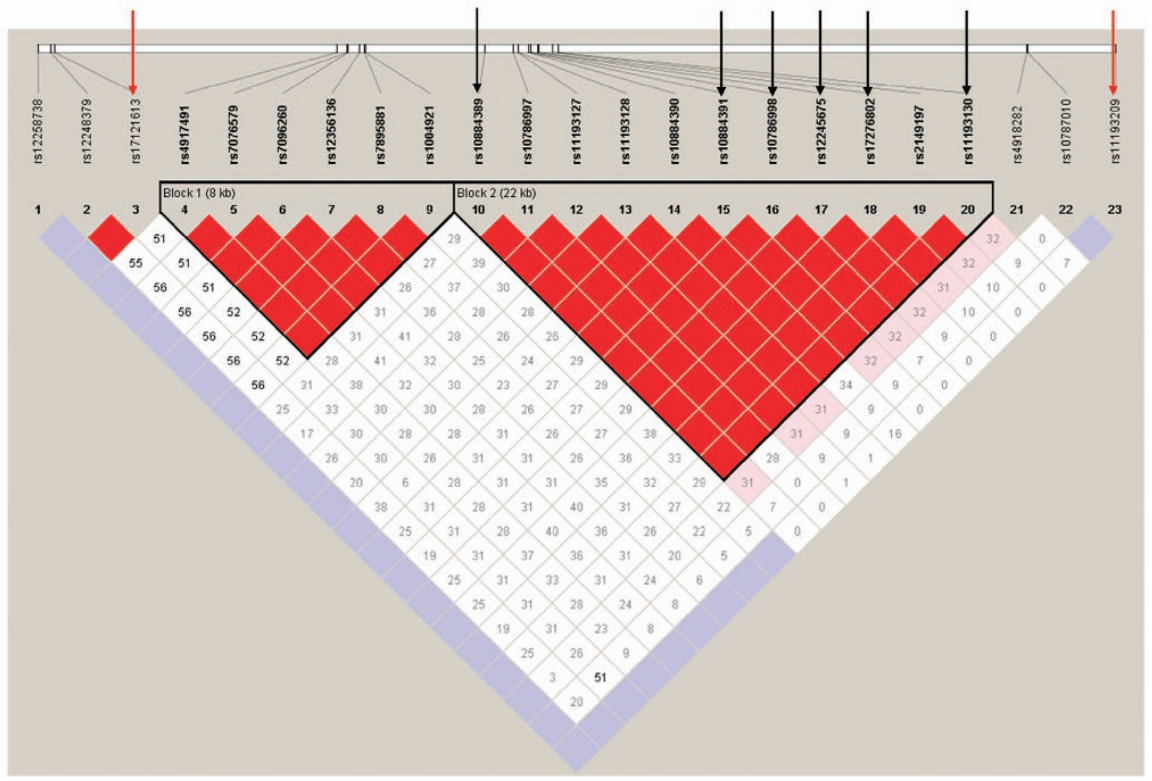

Figure 1 Black arrows: (a) The linkage disequilibrium (LD) block in sortilin-related VPS10 domain-containing receptor 3 (SORCS3) showing significant interaction with (b) two specific LD blocks in SORCS2 and (c) one specific LD block in SORCS1. Red arrows: Additional single nucleotide polymorphisms (SNPS) showing epistasis between SORCS2 and SORCS3. (a) The single LD block in SORCS3 showing epistasis with two specific LD blocks in SORCS2 (Figure 3b) and one specific LD block in SORCS1 (Figure 3c). (b) The two specific two LD blocks in SORCS2 (black arrows) showing epistasis with SORCS3, and SNPs showing in addition epistasis with SORCS1 (red arrows). (c) The specific LD block in SORCS1 (black arrows) showing epistasis with the specific LD block in SORCS3, and SNPs showing epistasis with SNPs in SORCS2 (red arrows). 

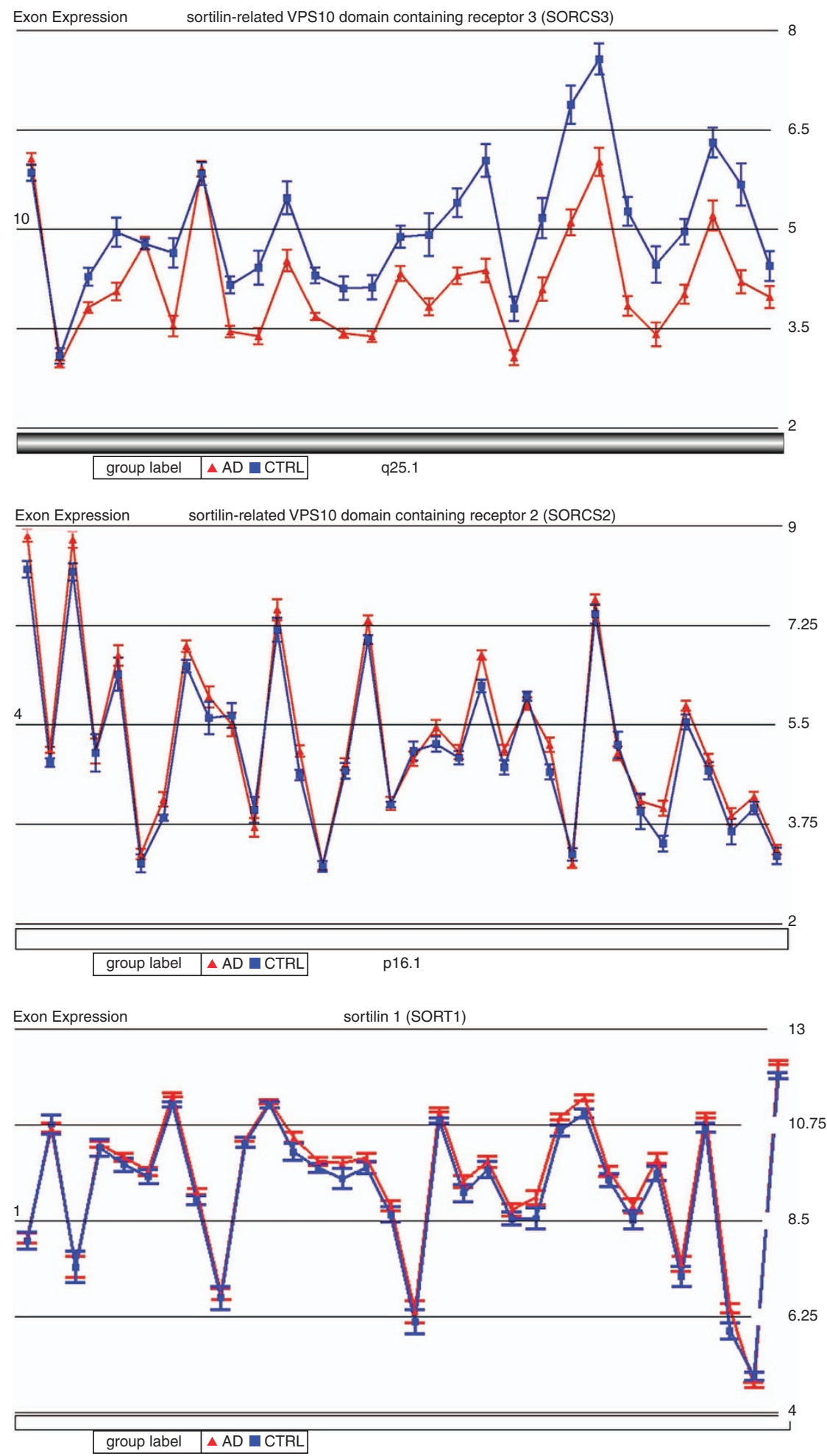

Figure 2 (a) View of sortilin-related VPS10 domain-containing receptor 3 (SORCS3) exon expression profile in 19 Alzheimer's disease (AD; red triangles) and 10 control (CTRL; blue squares) amygdala tissue. Each triangle dot represents least squares mean expression of an exon in $\mathrm{AD}$ tissue; each square dot represents least squares mean expression of an exon in control tissue. The mean gene expression intensity of AD vs controls was $4.17 \pm 0.43$ vs $5.03 \pm 0.49(P=5.1 \mathrm{E}-5)$ across all exons. (b) View of SORCS2 exon expression profile in $19 \mathrm{AD}$ (red triangles) and 10 control (blue squares) amygdala tissue. Each triangle dot represents least squares mean expression of an exon in $A D$ tissue; each square dot represents least squares mean expression of an exon in control tissue. The mean gene expression intensity of $A D$ vs controls was $5.29 \pm 0.33$ vs $5.08 \pm 0.32(P=0.12)$ across all exons. (c) View of sortilin 1 (SORT1) exon expression profile in $19 \mathrm{AD}$ (red triangles) and 10 control (blue squares) amygdala tissue. Each triangle dot represents least squares mean expression of an exon in $A D$ tissue; each square dot represents least squares mean expression of an exon in control tissue. The mean gene expression intensity of $\mathrm{AD}$ vs controls was $9.43 \pm 0.38$ vs $9.21 \pm 0.39(P=0.17)$ across all exons. 
introns 1 and 2 (Figure 1c) adjacent to the exons encoding the VPS10 domain. The epistasis $\beta$ for these SORCS1/SORCS3 and SORCS1/SORCS2 interactions ranged from -0.39 to 0.40 , again reflecting larger effects (additive) than the corresponding single-marker analyses $(-0.15<\beta<0.20)$. Although the $P$-values for epistatic effects just missed the multiple testing threshold of 0.0001 , the number of significant interactions was clearly higher than expected by chance (expected SORCS2/SORCS3 interactions: 10.8). Forest plots for the SNPs with epistatic effects or strongest individual associations with AD (rs7665496, rs6840423, rs7684383, rs4234804, rs1670036, rs1792124, rs12248379, rs13110208) are shown in Supplementary Figure S2.

Microarray gene expression and quantitative RT-PCR analyses. Microarray expression analyses showed lower expression of SorCS3 in AD brains compared with control brains (mean gene expression intensity: $4.17 \pm 0.43$ vs $5.03 \pm 0.49(P=5.1 \mathrm{E}-5$; Figure $2 \mathrm{a})$, in line with what we had previously observed in SORL1 and SORCS1.2,3 To validate the significant results of the Affymetrix array, we conducted a quantitative RT-PCR for the SORCS3 gene, using brain tissue from the amygdala region. Calculation of the fold change rate with the Relative Quantitation method of DataAssist software confirmed the results of the expression array for all the three investigated SORCS3 exons. Compared with the control samples, the AD samples showed significantly reduced expression of exons 10, 17 and 21 of SORCS3; 87\% $(P=0.012), 74 \% \quad(P=0.003)$ and $83 \%$ $(P=0.003)$, respectively (Supplementary Figure S3). Notably, these findings were also validated by comparison with publicly available gene expression results (188 cases, 176 controls, $P<0.0001$, http://labs.med.miami.edu/myers/ ). ${ }^{30}$ We did not find a significant difference between $A D$ and controls in the expression levels of SORCS2 or SORT1 (Figures $2 \mathrm{~b}$ and c). There was no significant difference in the expression levels between $A D$ and control brains in brain tissue from regions unaffected by the disease process (occipital lobe, cerebellum) for any of the homologs.

Cell culture and transfection. cDNA transfection of Vps10 family members in Hek 293 cells demonstrated a significant $(0.01 \leqslant P \leqslant 0.05)$ decrease of $\gamma$-secretase (APP-GV), whereas there was no effect on the AICD-GV translocation assay (Figure 3).

$\gamma$-Secretase processing. In HEK293 cells (Figure 4), 4/4 SorCS2-shRNAs, 3/3 SorC3-shRNAs and 2/3 SORT1shRNAs caused a significant increase greater than threefold in APP processing (from $P<0.001$ to $P<0.05$ ) as compared with the result with the scrambled shRNA (analysis of variance with Bonferroni correction) while not affecting the nuclear translocation of the control AICD-GV only-fragment.

\section{Discussion}

Taken together with previous studies, ${ }^{2,3}$ the findings here indicate that variants in SORL1, SORCS1, SORCS2 and SORCS3 of the VPS10-d receptor family are associated with AD risk. The results are consistent with previous studies

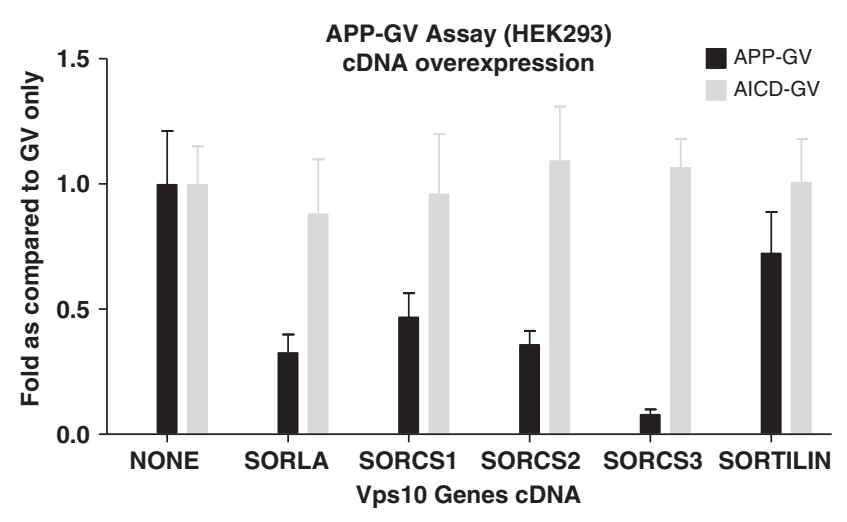

Figure 3 cDNA overexpression in transfected HEK293 cells. AICD, APP gene's C-terminus; APP, amyloid precursor protein; GV, GAL4 DNA-binding domain with VP16 transcriptional activator; SORCS, sortilin-related VPS10 domain-containing receptor; SORLA, sortilin-related receptor.

showing associations between the SNPS in SORL1 and SORCS1 with $A D^{2,3,11,13-16,18}$ and with cognitive performance. ${ }^{10}$ Similar to previous reports, the associated SNPs in SORCS1, SORCS2 and SORCS3 were mostly located in introns 1-3, implicating the VPS10 domain.

The effect sizes of the associated SNPs were small ( $\beta$ : -0.45 to 0.36$)$, but this is consistent with previous observations for the homologous genes SORL1 and $S O R C S 1^{2,3,18}$ as well as all recently detected novel AD susceptibility loci identified by large genome-wide association studies. $^{23,31-34}$

The epistasis models of SNPs significant in single-marker analyses further revealed pairwise SNP associations between specific LD blocks in the highly homologous SORCS1, SORCS2 and SORCS3. One single LD block in SORCS3 showed epistasis with both a single LD block in SORCS1 and two specific LD blocks in SORCS2. In addition, the same two regions of SORCS2 and SORCS3 interacted. Of note, the epistasis $\beta$ ranging from -0.94 to 0.94 reflected larger effects (additive) than the corresponding single-marker analyses $(-0.15<\beta<0.20)$, and the interacting SNPs are almost exclusively located in introns 1 and 2, adjacent to the exons encoding the VPS10 domain. This region has also been demonstrated to include the majority of disease-associated SNPs for both SORCS1 and SORL1.2,3,10,18,20 Our findings indicate that there are sequences within these specific LD blocks that are biologically important and that are interacting. The mechanism underlying these interactions is presently unclear. It could arise from direct interaction between the homologs, interactions with a mutual binding partner or interactions with a common substrate, such as APP or APP-CTF. However, it could also result from quite remote interactions that do not require a first- or second-order interaction between these proteins.

Suppression of SORCS2, SORCS3 or SORT1 increased $\gamma$-secretase processing of APP, findings consistent with reported effects by SORL1 and SORCS1 on $\gamma$-secretase processing of APP and changes in $A \beta 40$ and $A \beta 42$ levels. $^{2,3}$ Although SorCS3 and SorCS1 do not convey trans-Golgi network to late endosome sorting, ${ }^{35,36}$ SORT1 is - similar to SORL1-also capable of mediating sorting of ligands from the trans-Golgi network to late endosomes or lysosomes. ${ }^{22}$ 

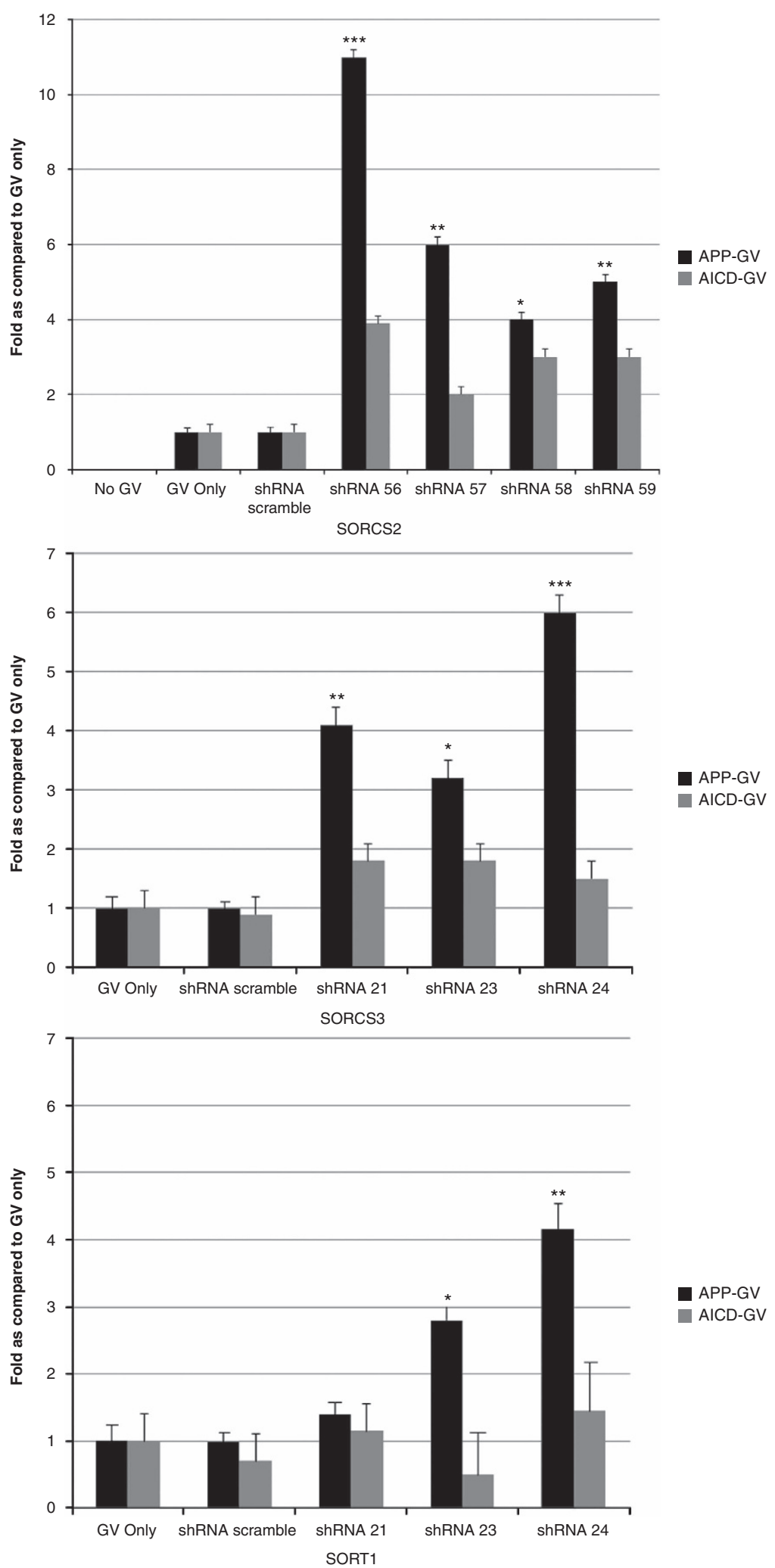

APP-GV

AICD-GV

Figure $4 \gamma$-Secretase activity and nuclear translocation of amyloid precursor protein (APP) assays with sortilin-related VPS10 domain-containing receptor 2 (SORCS2), SORCS3 or SORT1 short hairpin RNAs (shRNAs). Both the APP-GV (GAL4 DNA-binding domain with VP16 transcriptional activator) and APP gene's C-terminus (AICD)-GV assay were performed in HEK293 cells. The data from SorCS2, SorCS3 or sortilin 1 (SORT1) shRNA was normalized to either APP-GV only or AICD-GV with the scrambled sequence shRNA (shRNA-scrambled), which was included as a negative control. The data are representative for the assays, were performed in $\geqslant 3$ experiments in replicates of eight samples per condition (96-well format, s.d. bars are shown, ${ }^{\star} P<0.05,{ }^{\star \star} P<0.01,{ }^{\star \star \star} P<0.001$ as compared with APP-GV only (analysis of variance, Bonferroni's correction). 
Interestingly, sortilin-mediated endocytosis has been shown to determine levels of progranulin involved in frontotemporal dementia. ${ }^{37}$ Results from the current genome-wide association studies suggest that other genes PICALM, BIN1 and $C D 2 A P$, modulate intracellular trafficking of cell surface proteins. Thus, it is appears that in addition to their effect on $\gamma$ secretase processing of APP, some members of the VPS10-d receptor family exert their effect on $A D$ through modulation of APP trafficking.

Reduced expression of SORCS3 may be a secondary effect of the disease, but it is consistent with the $\gamma$-secretase assays, which indicated that suppression of SORCS3 activates $A \beta$ production. If correct, this would provide a potential explanation for how downregulation of SORCS3 might increase risk for $A D$. We are unable to see any difference in the expression of SORCS2 and SORT1. However, we cannot yet exclude the possibility that this was the result of the small sample size or chosen phenotype. In a previous study by Mufson et al., ${ }^{38}$ SORT1 levels wereconsistent with our findings-not associated with clinical diagnosis or antemortem cognitive test scores. However, there was an association with severity of neuropathology by Braak and NIA-Reagan diagnoses.

The significant strengths of this study are the large sample size, allowing us to detect small effects and explore epistasis. Limitations include that the SNPs assessed were derived from available genome-wide arrays. Thus, they do not cover the complete genetic variation in these genes, and it is possible that there are additional disease-associated markers that have not been genotyped. It is also possible that we lacked the power to detect additional disease-associated markers or interactions of SNPs with lower allele frequencies or effect sizes.

Taken together, our results indicate that in addition to SORL1 and SORCS1, the variants in other members of the VPS 10-D receptor family (SORCS2, SORCS3 and SORT1) are associated with $A D$ either independently or through epistatic mechanisms.

\section{Conflict of interest}

The authors declare no conflict of interest.

Acknowledgements. The National Institutes of Health, National Institute on Aging (NIH-NIA) supported this work through the following grants: ADGC, U01AG032984, RC2AG036528;NACC, U01AG016976; NCRAD, U24AG021886; NIA LOAD, U24 AG026395,U24AG026390; Boston University, P30 AG013846, R01 HG02213, K24 AG027841, U01 AG10483, R01 CA129769, R01 MH080295,R01 AG009029, R01 AG017173, R01 AG025259; Columbia University, P50 AG008702, R37 AG015473; Duke University, P30 AG028377; Emory University, AG025688; Indiana University, P30 AG10133; Johns Hopkins University, P50 AG005146,R01 AG020688; Massachusetts General Hospital, P50 AG005134 Mayo Clinic, P50 AG016574; Mount Sinai School of Medicine, P50 AG005138, P01 AG002219; New York University, P30 AG08051, M01RR00096 and UL1 RR029893; Northwestern University, P30AG013854; Oregon Health and Science University, P30AG008017,R01 AG026916; Rush University, P30AG010161,R01AG019085,R01AG15819, R01AG17917, R01AG30146; University of Alabama at Birmingham, P50 AG016582, UL1RR02777; University of Arizona/TGEN, P30 AG019610,R01 AG031581,R01 NS059873; University of California, Davis, P30 AG010129; University of California, Irvine, P50AG016573, P50, P50AG016575, P50AG016576, P50AG016577; University of California, Los Angeles, P50AG016570; University of California, San Diego, P50AG005131; University of
California, San Francisco, P50AG023501, P01AG019724; University of Kentucky, P3OAG028383; University of Michigan, P50AG008671; University of Pennsylvania, P30AG010124; University of Pittsburgh, P50AG005133,AG030653; University of Southern California, P50AG005142; University of Texas Southwestern, P30AG012300; University of Miami, R01 AG027944, AG010491, AG027944, AG021547, AG019757; University of Washington, P50 AG005136, UO1 AG06781, U01 HG004610; Vanderbilt University, R01 AG019085; and Washington University, P50 AG005681, P01 AG03991. ADNI Funding for ADNI is through the Northern California Institute for Research and Education by grants from Abbott, AstraZeneca $A B$, Bayer Schering Pharma AG, Bristol-Myers Squibb, Eisai Global Clinical Development, Elan Corporation, Genentech, GE Healthcare, GlaxoSmithKline, Innogenetics, Johnson and Johnson, Eli Lilly and Co., Medpace, Inc., Merck and Co., Inc., Novartis AG, Pfizer Inc, F. Hoffman-La Roche, Schering-Plough, Synarc, Inc., Alzheimer's Association, Alzheimer's Drug Discovery Foundation, the Dana Foundation, and by the National Institute of Biomedical Imaging and Bioengineering and NIA grants U01 AG024904, RC2 AG036535, K01 AG030514. We thank Creighton Phelps, Steven Synder and Marilyn Miller from NIA who are ex-officio ADGC members. Support was also from the Alzheimer's Association (LAF, IIRG-0889720; MP-V, IIRG-05-14147) and the Veterans Affairs Administration. PSG-H is supported by Wellcome Trust, Howard Hughes Medical Institute, and the Canadian Institute of Health Research. This work was also supported by the Evans Center for Interdisciplinary Biomedical Research ARC on 'Protein Trafficking and Neurodegenerative Diseases' at Boston University (http://www.bumc.bu.edu/ evanscenteribr/) Canadian Institutes of Health Research, Ontario Research Fund $(E R, P H)$, the Alzheimer Society of Canada, the Alzheimer Society of Ontario, Howard Hughes Medical Institute, and The Wellcome Trust (PH). CR was further supported by a Paul B. Beeson Career Development Award (K23AG034550). For the ADGC, biological samples and associated phenotypic data used in primary data analyses were stored at Principal Investigators' institutions and at the National Cell Repository for Alzheimer's Disease (NCRAD) at Indiana University funded by NIA. Associated phenotypic data used in secondary data analyses were stored at NCRAD and at the National Institute on Aging Alzheimer's Disease Data Storage Site (NIAGADS) at the University of Pennsylvania, funded by NIA. Contributors to the Genetic Analysis Data included Principal Investigators on projects that were individually funded by NIA, other NIH institutes or private entities.

1. Selkoe DJ. Amyloid beta protein precursor and the pathogenesis of Alzheimer's disease. Cell 1989; 58: 611-612

2. Reitz C, Tokuhiro S, Clark LN, Conrad C, Vonsattel JP, Hazrati LN et al. SORCS1 alters amyloid precursor protein processing and variants may increase Alzheimer's disease risk. Ann Neurol 2011; 69: 47-64.

3. Rogaeva E, Meng Y, Lee JH, Gu Y, Kawarai T, Zou F et al. The neuronal sortilin-related receptor SORL1 is genetically associated with Alzheimer disease. Nat Genet 2007; 39: 168-177.

4. Liang X, Slifer M, Martin ER, Schnetz-Boutaud N, Bartlett J, Anderson B et al. Genomic convergence to identify candidate genes for Alzheimer disease on chromosome 10. Human Mutat 2009; 30: 463-471.

5. Hermey G, Riedel IB, Hampe W, Schaller HC, Hermans-Borgmeyer I. Identification and characterization of SorCS, a third member of a novel receptor family. Biochem Biophys Res Commun 1999; 266: 347-351.

6. Jacobsen L, Madsen P, Moestrup SK, Lund AH, Tommerup N, Nykjaer A et al. Molecular characterization of a novel human hybrid-type receptor that binds the alpha2macroglobulin receptor-associated protein. J Biol Chem 1996; 271: 31379-31383.

7. Kikuno R, Nagase T, Ishikawa K, Hirosawa M, Miyajima N, Tanaka A et al. Prediction of the coding sequences of unidentified human genes. XIV. The complete sequences of 100 new cDNA clones from brain which code for large proteins in vitro. DNA Res 1999; 6: 197-205.

8. Rezgaoui M, Hermey G, Riedel IB, Hampe W, Schaller HC, Hermans-Borgmeyer I. Identification of SorCS2, a novel member of the VPS10 domain containing receptor family, prominently expressed in the developing mouse brain. Mech Dev 2001; 100: 335-338.

9. Petersen CM, Nielsen MS, Nykjaer A, Jacobsen L, Tommerup N, Rasmussen $\mathrm{HH}$ et al. Molecular identification of a novel candidate sorting receptor purified from human brain by receptor-associated protein affinity chromatography. J Biol Chem 1997; 272: 3599-3605.

10. Reitz C, Lee JH, Rogers RS, Mayeux R. Impact of genetic variation in SORCS1 on memory retention. PLoS One 2011; 6: e24588.

11. Bettens K, Brouwers N, Engelborghs S, De Deyn PP, Van Broeckhoven C, Sleegers K. SORL1 is genetically associated with increased risk for late-onset Alzheimer disease in the Belgian population. Human Mutat 2008; 29: 769-770.

12. Cellini E, Tedde A, Bagnoli S, Pradella S, Piacentini S, Sorbi S et al. Implication of sex and SORL1 variants in italian patients with Alzheimer disease. Arch Neurol 2009; 66: 1260-1266. 
13. Cuenco KT, Lunetta KL, Baldwin CT, McKee AC, Guo J, Cupples LA et al. Association of distinct variants in SORL1 with cerebrovascular and neurodegenerative changes related to Alzheimer disease. Arch Neurol 2008; 65: 1640-1648.

14. Grupe A, Li Y, Rowland C, Nowotny P, Hinrichs AL, Smemo S et al. A scan of chromosome 10 identifies a novel locus showing strong association with late-onset Alzheimer disease. Am J Human Genet 2006; 78: 78-88.

15. Kimura R, Yamamoto M, Morihara T, Akatsu H, Kudo T, Kamino K et al. SORL1 is genetically associated with Alzheimer disease in a Japanese population. Neurosci Lett 2009; 461: 177-180.

16. Kolsch $\mathrm{H}$, Jessen $\mathrm{F}$, Wiltfang J, Lewczuk $P$, Dichgans M, Teipel SJ et al. Association of SORL1 gene variants with Alzheimer's disease. Brain Res 2009; 1264: 1-6.

17. Meng Y, Lee JH, Cheng R St, George-Hyslop P, Mayeux R, Farrer LA. Association between SORL1 and Alzheimer's disease in a genome-wide study. Neuroreport 2007; 18: $1761-1764$.

18. Reitz C, Cheng R, Rogaeva E, Lee JH, Tokuhiro S, Zou F et al. Meta-analysis of the association between variants in SORL1 and Alzheimer disease. Arch Neurol 2011; 68: 99-106.

19. Lane RF, Raines SM, Steele JW, Ehrlich ME, Lah JA, Small SA et al. Diabetes-associated SorCS1 regulates Alzheimer's amyloid-beta metabolism: evidence for involvement of SorL1 and the retromer complex. J Neurosci 2010; 30: 13110-13115.

20. Hermey G, Plath N, Hubner CA, Kuhl D, Schaller HC, Hermans-Borgmeyer I. The three sorCS genes are differentially expressed and regulated by synaptic activity. $J$ Neurochem 2004; 88: 1470-1476.

21. Schmidt V, Baum K, Lao A, Rateitschak K, Schmitz Y, Teichmann A et al. Quantitative modelling of amyloidogenic processing and its influence by SORLA in Alzheimer's disease. EMBO J 2012; 31: 187-200.

22. Hermey G. The Vps10p-domain receptor family. Cell Mol Life Sci 2009; 66: 2677-2689.

23. Naj AC, Jun G, Beecham GW, Wang LS, Vardarajan BN, Buros J et al. Common variants at MS4A4/MS4A6E, CD2AP, CD33 and EPHA1 are associated with late-onset Alzheimer's disease. Nat Genet 2011; 43: 436-441.

24. Cao X, Sudhof TC. Dissection of amyloid-beta precursor protein-dependent transcriptional transactivation. J Biol Chem 2004; 279: 24601-24611.

25. Lewandowski NM, Small SA. Brain microarray: finding needles in molecular haystacks. J Neurosci 2005; 25: 10341-10346.

26. Carey VJ. GEE: Generalized Estimation Equation Solver. 4.13-15 edn 2010.

27. Zeger SL, Liang KY, Albert PS. Models for longitudinal data: a generalized estimating equation approach. Biometrics 1988; 44: 1049-1060.
28. Li J, Ji L. Adjusting multiple testing in multilocus analyses using the eigenvalues of a correlation matrix. Heredity (Edinb) 2005; 95: 221-227.

29. Schmittgen TD, Livak KJ. Analyzing real-time PCR data by the comparative C(T) method. Nat Protoc 2008; 3: 1101-1108.

30. Webster JA, Gibbs JR, Clarke J, Ray M, Zhang W, Holmans P et al. Genetic control of human brain transcript expression in Alzheimer disease. Am J Human Genet 2009; 84: 445-458.

31. Harold D, Abraham R, Hollingworth P, Sims R, Gerrish A, Hamshere ML et al. Genomewide association study identifies variants at CLU and PICALM associated with Alzheimer's disease. Nat Genet 2009; 41: 1088-1093.

32. Hollingworth $P$, Harold D, Sims R, Gerrish A, Lambert JC, Carrasquillo MM et al. Common variants at $A B C A 7, M S 4 A 6 A / M S 4 A 4 E, E P H A 1, C D 33$ and CD2AP are associated with Alzheimer's disease. Nat Genet 2011; 43: 429-435.

33. Lambert JC, Heath S, Even G, Campion D, Sleegers K, Hiltunen M et al. Genome-wide association study identifies variants at CLU and CR1 associated with Alzheimer's disease. Nat Genet 2009; 41: 1094-1099.

34. Seshadri S, Fitzpatrick AL, Ikram MA, DeStefano AL, Gudnason V, Boada M et al. Genome-wide analysis of genetic loci associated with Alzheimer disease. JAMA 2010; 303: 1832-1840.

35. Westergaard UB, Kirkegaard K, Sorensen ES, Jacobsen C, Nielsen MS, Petersen CM et al. SorCS3 does not require propeptide cleavage to bind nerve growth factor. FEBS Lett 2005; 579: 1172-1176

36. Nielsen MS, Keat SJ, Hamati JW, Madsen P, Gutzmann JJ, Engelsberg A et al. Different motifs regulate trafficking of SorCS1 isoforms. Traffic 2008; 9: 980-994.

37. Hu F, Padukkavidana T, Vaegter CB, Brady OA, Zheng Y, Mackenzie IR et al. Sortilinmediated endocytosis determines levels of the frontotemporal dementia protein, progranulin. Neuron 2010; 68: 654-667.

38. Mufson EJ, Wuu J, Counts SE, Nykjaer A. Preservation of cortical sortilin protein levels in $\mathrm{MCl}$ and Alzheimer's disease. Neurosci Lett 2010; 471: 129-133.

(i) (-) $\odot$ Translational Psychiatry is an open-access journal published by Nature Publishing Group. This work is licensed under a Creative Commons Attribution-NonCommercialNoDerivs 3.0 Unported License. To view a copy of this license, visit http://creativecommons.org/licenses/by-nc-nd/3.0/

Supplementary Information accompanies the paper on the Translational Psychiatry website (http://www.nature.com/tp) 